\title{
Determinação dos limiares auditivos de forma objetiva e automática em adultos
}

\section{normo-ouvintes}

Objective and automatic determination of hearing thresholds in normal hearing adult subjects

Determinación objetiva y automática de los umbrales auditivos en adultos con audición normal

Recebido: 21/09/2021 | Revisado: 01/10/2021 | Aceito: 05/10/2021 | Publicado: 09/10/2021

Dayane Stephanie Potgurski

ORCID: https://orcid.org/0000-0001-8646-6271 Universidade Federal de Santa Catarina, Brasil

E-mail: dayanepotgurski@gmail.com

Georgea Espindola Ribeiro

ORCID: https://orcid.org/0000-0003-3969-5252

Universidade de São Paulo, Brasil

E-mail: georgea_espindola@hotmail.com

Daniela Polo Camargo da Silva

ORCID: https://orcid.org/0000-0003-2082-9361

Universidade Federal de Santa Catarina, Brasil

E-mail: daniela.polo@ufsc.br

\begin{abstract}
Resumo
Objetivo: verificar a aplicabilidade do exame de resposta auditiva de estado estável (RAEE), correlacionando os limiares auditivos obtidos neste exame com os limiares auditivos obtidos na audiometria tonal liminar (ATL) em indivíduos sem queixas auditivas. Metodologia: Foram avaliados 15 indivíduos adultos normo-ouvintes. A avaliação foi composta pela ATL e RAEE, ambas para verificação do grau da audição, sendo a primeira uma medida comportamental e a segunda, eletrofisiológica. Na ATL foram avaliadas a acuidade auditiva nas frequências de 250, 500, 1000, 2000, 3000, 4000, 6000 e $8000 \mathrm{~Hz}$, de ambas as orelhas. A pesquisa da RAEE foi realizada em modo multifrequencial e dicótico, ou seja, foram pesquisas as frequência de 500, 1000, 2000 e $4000 \mathrm{~Hz}$ nas duas orelhas ao mesmo tempo, com o objetivo de avaliar o nível mínimo de resposta obtido de maneira automática. Resultados: Na ATL os limiares obtidos estiveram abaixo de 25 dBNA para todas as frequências avaliadas e a mediana dos valores encontrados foram menores do que os valores medianos encontrados na RAEE. No teste de correlação de Spearman, houve correlação significativa apenas na frequência de $500 \mathrm{~Hz}$. Conclusão: Os limiares auditivos obtidos pela RAEE foram detectados em todos os participantes do estudo e os valores foram superiores aos encontrados na ATL, entretanto é comum os resultados eletrofisiológicos serem superiores aos observados em exames comportamentais. Não houve correlação entre os limiares obtidos entre as técnicas para a maioria das frequências avaliadas, em indivíduos adultos normo-ouvintes. Desta forma, sugere-se a realização de pesquisas futuras com um maior tamanho amostral, adotando-se a mesma metodologia aplicada nesse estudo.
\end{abstract}

Palavras-chave: Potenciais evocados auditivos; Audição; Adulto.

\begin{abstract}
Objective: to verify the applicability of the auditory steady state response (ASSR) test, correlating the auditory thresholds obtained in this test with the pure tone audiometry (PTA) in those without hearing complaints. Methodology: fifteen normal hearing adults were registered. The evaluation consisted of the PTA and ASSR, both to verify the degree of hearing, the first being a behavioral measure and the second, electrophysiological. In PTA, the hearing threshold was evaluated in the frequencies of 250, 500, 1000, 2000, 3000, 4000, 6000 and $8000 \mathrm{~Hz}$, in both ears. The ASSR survey was carried out in multifrequency and dichotic mode, that is, surveys with frequencies of 500, 1000,2000 and $4000 \mathrm{~Hz}$ in both ears at the same time, with the aim of automatically evaluating the minimum level of specified response. Results: in PTA, the hearing thresholds were below $25 \mathrm{dBHL}$ for all evaluated frequencies and the median values found were lower than the median values found in ASSR. Test Spearman, there was a significant correlation only in the frequency of $500 \mathrm{~Hz}$. Conclusion: the hearing thresholds obtained by ASSR were detected in all study participants and the values were higher than those found in the ATL, however it is common for the electrophysiological results to be higher than those observed in behavioral tests. There was no correlation between the thresholds obtained between the techniques for most frequencies evaluated in normal hearing adult individuals. Thus, it is suggested to carry out future research with a larger sample size, adopting the same methodology applied in this study.
\end{abstract}

Keywords: Evoked potentials; Hearing; Adult. 


\begin{abstract}
Resumen
Objetivo: verificar la aplicabilidad de la Prueba de Respuesta Auditiva en Estado Estacionario (RAEE), correlacionando los umbrales auditivos obtenidos en esta prueba con los umbrales auditivos obtenidos en audiometría de tonos puros (ATP) en personas sin quejas de audición. Metodología: se evaluaron quince individuos adultos con audición normal. La evaluación consistió en el ATP y RAEE, ambos para verificar el grado de audición. En ATP se evaluó la agudeza auditiva en las frecuencias de 250, 500, 1000, 2000, 3000, 4000, 6000 y 8000 Hz, en ambos oídos. La investigación RAEE se realizó en modo multifrecuencia y dicótico, es decir, se investigaron las frecuencias de 500, 1000, 2000 y $4000 \mathrm{~Hz}$ en ambos oídos al mismo tiempo. Resultados: en el ATP, los umbrales obtenidos fueron inferiores a $25 \mathrm{dBHL}$ para todas las frecuencias evaluadas y los valores medianos encontrados fueron inferiores a los valores medianos encontrados en el RAEE. En la prueba de correlación de Spearman, hubo una correlación significativa solo a la frecuencia de $500 \mathrm{~Hz}$. Conclusión: los umbrales de audición obtenidos por RAEE se detectaron en todos los participantes del estudio y los valores fueron superiores a los encontrados en el ATP, sin embargo es común para que los resultados electrofisiológicos sean superiores a los observados en las pruebas de comportamiento. No hubo correlación entre los umbrales obtenidos entre las técnicas para la mayoría de las frecuencias evaluadas en adultos con audición normal. Así, se sugiere realizar futuras investigaciones con un tamaño muestral mayor, adoptando la misma metodología aplicada en este estudio.
\end{abstract}

Palabras clave: Potenciales evocados auditivos; Audición; Adulto.

\title{
1. Introdução
}

A Audiometria Tonal Liminar (ATL) é considerada o exame padrão ouro para o diagnóstico audiológico. Este é um procedimento comportamental que determina a frequência e a intensidade com que o sujeito pode detectar os sons, sendo assim, fundamental para determinar os limiares auditivos ao comparar os valores obtidos com os padrões da normalidade. É por meio da ATL que se possibilita definir a presença da deficiência auditiva, bem como caracterizá-la quanto ao tipo, grau e configuração audiométrica (Lins et al., 1996; Korczac et al., 2012).

Este exame é de extrema importância, uma vez que permite determinar o topodiagnóstico da lesão e inferir seu comprometimento no desempenho comunicativo do sujeito. Além disso, a ATL possibilita auxílio para o diagnóstico diferencial das lesões em todas as regiões da orelha, fornece informações para o estabelecimento da causa e reabilitação da deficiência auditiva, opções de tratamento e determina a necessidade de encaminhamento para atendimento médico (Lins et al., 1996; D’Haenens et al., 2008; Korczac et al., 2012).

Outra técnica que permite verificar estruturas da via auditiva são os potenciais evocados auditivos, que avaliam a atividade elétrica do nervo auditivo até o córtex cerebral em resposta a um estímulo acústico, refletindo os caminhos elétricos gerados por neurônios de diferentes fibras, tratos ou núcleos do sistema nervoso central. Diferente dos exames auditivos comportamentais, os potenciais são realizados de forma objetiva, não sendo necessária a participação do indivíduo avaliado (D’Haenens et al., 2008)3.

Dentre os potenciais evocados auditivos existentes, a resposta auditiva de estado estável (RAEE) é um deles, trata-se de um procedimento eletrofisiológico que permite verificar a audição de forma automática, realizando a estimulação das vias auditivas até níveis próximos a 130 dBNA. Sendo assim, pode-se medir a audição residual e prescrever o ganho necessário para a prótese auditiva. Além disso, este exame contribui na avaliação audiológica de sujeitos que apresentem dificuldade em responder aos testes auditivos comportamentais (D’Haenens et al., 2008; Bucuvic \& Iório, 2011).

Em determinadas situações, quando o indivíduo não consegue realizar a ATL, por limitações cognitivas, por falta de colaboração, para fins legais ou quando estamos diante de uma população infantil, a RAEE tem adquirido espaço importante na bateria de exames auditivos (Bucuvic \& Iório, 2011).

Alguns estudos avaliaram a confiabilidade da RAEE em determinar os limiares auditivos em frequência específica para estabelecer uma correlação com os limiares obtidos na ATL, além de apontarem outras vantagens como a detecção objetiva do limiar e avaliação simultânea de múltiplas frequências (Valdes et al., 1997; John \& Picton, 2000). No entanto, ainda há escassez de publicações que abordam essa temática principalmente a nível nacional, o que torna necessário investigar 
o padrão de resposta de adultos normo-ouvintes para a prática clínica, comparando os resultados obtidos na RAEE com a ATL.

Assim, o objetivo deste estudo foi verificar a aplicabilidade do exame de RAEE, correlacionando os limiares auditivos obtidos neste exame com os limiares auditivos obtidos na ATL em indivíduos sem queixas auditivas.

\section{Metodologia}

- Participantes

Tratou-se de um estudo de corte transversal (Estrela, 2018) que avaliou 15 adultos normo-ouvintes ( $\mathrm{n}=30$ orelhas), de ambos os gêneros, entre 19 a 40 anos. O estudo foi conduzido entre março de 2020 a setembro de 2021 . Todos os participantes foram informados sobre a finalidade do estudo e assinaram o Termo de Consentimento Livre e Esclarecido, de acordo com a aprovação pelo Comitê de Ética em Pesquisa com Seres Humanos sob o número CAAE: 97184718.4.0000.0121.

Critérios de inclusão: nenhuma queixa otológica ou auditiva, ATL com limiares iguais ou menores que 25 dBNA nas frequências de 250, 500, 1000, 2000, 3000, 4000, 6000 e $8000 \mathrm{~Hz}$.

Critérios de exclusão: obstrução por cera no conduto observado por meio de metatoscopia, qualquer alteração audiológica identificada na ATL ou alterações neurológicas.

- Avaliação Audiológica

A ATL foi realizada em cabine acústica com o audiômetro Itera II (Madsen) calibrado segundo o padrão ANSI S3.61996. Os limiares auditivos por via aérea foram obtidos com os tons puros nas frequências de 250, 500, 1000, 2000, 3000, 4000, 6000 e $8000 \mathrm{~Hz}$, pelo método descendente, ou seja, do som para o silêncio bilateralmente.

A RAEE foi feita no equipamento Smart (Intelligent Hearing System). Após a limpeza da pele com substância abrasiva (Nuprep®), os eletrodos de superfície, Ambu®Neuroline 720 00S (Dinamarca), foram fixados em pontos específicos. O eletrodo positivo (ativo) foi fixado à fronte (Fz) e os negativos (de referência) às regiões da mastoide (M1 e M2). O eletrodo terra (neutro) foi colocado na fronte (Fpz). O estímulo utilizado consistiu da combinação múltipla simultânea de quatro tone pipes portadores das frequências de 500,1000, 2000 e $4000 \mathrm{~Hz}$, nas frequências de modulação de aproximadamente 77, 85, 93 e $101 \mathrm{~Hz}$ na orelha esquerda e de 79, 87, 95 e $103 \mathrm{~Hz}$ na orelha direita respectivamente. Foi pesquisado o nível mínimo de resposta (NMR) das frequências de 500, 1000, 2000 e $4000 \mathrm{~Hz}$ simultaneamente em ambas as orelhas. Os NMR foram pesquisados a passos de $10 \mathrm{~dB}$. Conforme as respostas eram observadas nas frequências pesquisadas, com ruído elétrico menor que $0,05 \mu \mathrm{V}$, a análise era interrompida na frequência a qual encontrou-se o NMR e a pesquisa nas demais frequências era reiniciada. Cada estímulo múltiplo simultâneo foi apresentado bilateralmente através de fones de inserção ER-3A. A intensidade inicial pesquisada foi de $60 \mathrm{~dB}$ e não ultrapassou 80 dBNPS. O número máximo de estímulos foi ajustado para 400 com duração de 1,024 milissegundos cada, divididos em 20 varreduras. As amostras do eletroencefalograma (EEG) após uma varredura foram filtradas com filtro de 30-3000 Hz, e amplificadas com um ganho de $1000 \mathrm{~K}$, sendo em seguida processadas utilizando uma taxa de conversão A/D de $20 \mathrm{kHz}$. Após cada varredura a Transformação Rápida de Fourier (FFT) foi realizada automaticamente pelo software, mostrando o resultado obtido em um lote polar e em um espectro de frequências. $\mathrm{O}$ teste $\mathrm{F}$ calculou a probabilidade da amplitude da resposta ser significativamente diferente da amplitude média do ruído de fundo na frequência de modulação, assim como da amplitude média do ruído de fundo nas frequências adjacentes. Se a relação sinalruído foi maior que $6,13 \mathrm{~dB}(\mathrm{p}=0,05)$ nas duas condições, o sinal foi considerado uma resposta.

Os resultados da RAEE foram convertidos de dBNPS para dBNA de acordo com a norma ISO 389-2 para fones de inserção, com as correções de -6, -0, -3 e -6 dB respectivamente para as frequências de 500, 1000, 2000 e $4000 \mathrm{~Hz}$. 
- Análise dos resultados

Os resultados não apresentaram distribuição normal dos dados, assim para a comparação dos limiares obtidos nos exames de ATL e RAEE entre as orelhas foi utilizado o teste T pareado, os resultados foram apresentados em mediana, primeiro e terceiro quartis. Para a análise de correlação entre os resultados da RAEE e ATL foi analisada a estimativa da correlação de Spearman. A análise foi feita com o software Jamovi, versão 1.6.23.

\section{Resultados}

Foram analisados os resultados da ATL e RAEE em 15 indivíduos adultos, sendo seis do sexo feminino nove do sexo masculino.

A análise separada das orelhas direita e esquerda não demonstrou diferença significativa dos limiares entre elas (teste T pareado), nos exames de ATL e RAEE, assim para a comparação entre os resultados dos limiares obtidos entre esses exames foram considerados em conjunto $(n=30)$.

Na ATL a mediana (primeiro e terceiro quartis) dos limiares auditivos obtidos para todas as frequências de 500, 1000, 2000 e $4000 \mathrm{~Hz}$ foram de: 5 dBNA (5 - 10), 5 (0 - 5), 5 (0 - 5), 5 (0 - 5), respectivamente.

Dentre os indivíduos avaliados, foram obtidos limiares mensuráveis em $100 \%$ das frequências testadas na RAEE. Assim, na RAEE a mediana (primeiro e terceiro quartis) dos limiares auditivos obtidos para todas as frequências de 500, 1000, 2000 e $4000 \mathrm{~Hz}$ foram de: 24 dBNA (14 - 24), 24 (19 - 29), 17 (7- 24), 11 (11 - 21), respectivamente.

$\mathrm{Na}$ análise de correlação entre os limiares auditivos obtidos nas frequências de 500, 1000, 2000 e $4000 \mathrm{~Hz}$ nos exames de RAEE e ATL, a única frequência que apresentou correlação significativa foi a de $500 \mathrm{~Hz}$ ( $\mathrm{p}=0,045 ; \mathrm{r}=-0,368$ ), que mostra que quanto maior foi o valor do limiar obtido na RAEE, menor foi o valor do limiar obtido da ATL (correlação de Speaman) (Figura 1).

Figura 1. Correlação entre os limiares auditivos de 500, 1000, 2000 e $4000 \mathrm{~Hz}$ entre os exames de RAEE e ATL (Correlação de Spearman).

Figura 1. Correlação entre os limiares auditivos de 500, 1000, 2000 e $4000 \mathrm{~Hz}$ entre os exames de RAEE e ATL (Correlação de Spearman).
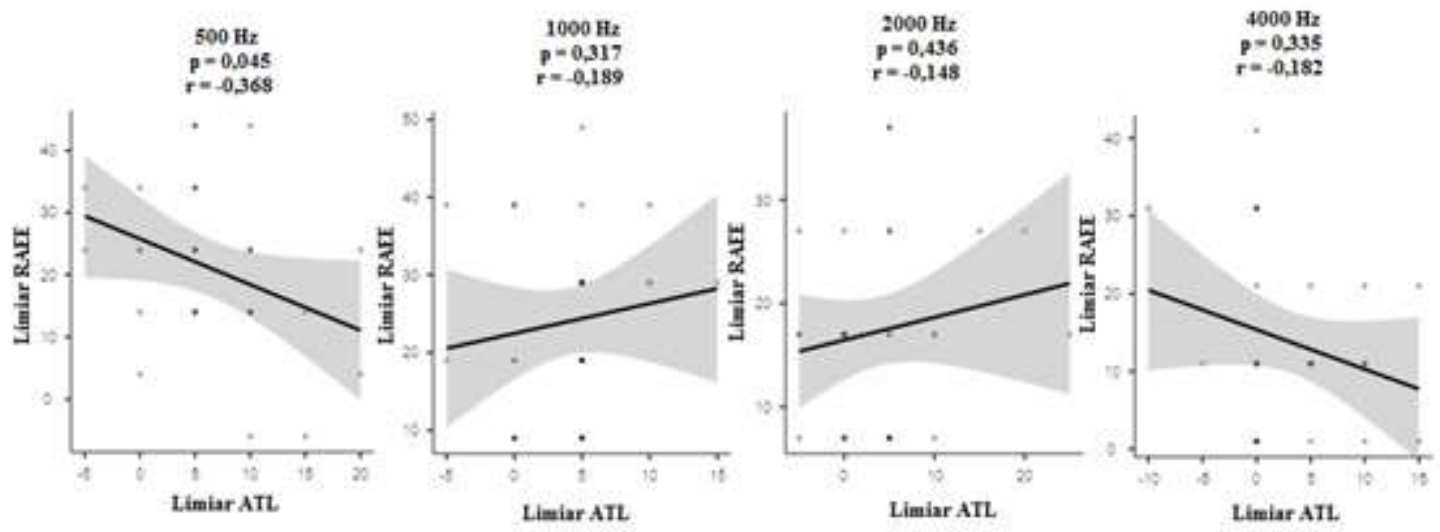

Fonte: Autores.

\section{Discussão}

A determinação da acuidade auditiva é extremamente importante na determinação da conduta a ser realizada, principalmente quando há prejuízo da capacidade de ouvir (Bucuvic \& Iório, 2011; Dabbous et., 2019). 
A pesquisa dos limiares auditivos de forma objetiva, por medidas eletrofisiológicas é útil em casos difíceis de serem avaliados, para fins legais e na população pediátrica, que ainda não apresenta respostas precisas por medidas comportamentais, nos primeiros anos de vida. Assim, verificar se as técnicas existentes apresentam resultados confiáveis é necessário (Lins et al., 1996; D’Haenens et al., 2008; Bucuvic \& Iório, 2011; Korczac et al., 2012; Dabbous et al., 2017).

Esta pesquisa foi realizada apenas com indivíduos adultos normo-ouvintes para verificar com precisão se os resultados obtidos por medidas comportamentais, na ATL, são compatíveis com os resultados obtidos por medidas eletrofisiológicas, por meio da RAEE. Desta forma, em situações onde apenas a medida eletrofisiológica seja possível a conduta após o resultado obtido deverá ser a mesma.

Verificou-se que os limiares auditivos obtidos por meio da ATL são melhores aos obtidos na RAEE, ou seja, os valores são menores quando determinados com a medida subjetiva do que com a medida objetiva, além de ser encontrada menor variabilidade entre as respostas quando obtidas por meio da ATL, tais dados são compatíveis aos descritos na literatura (Ferraz et al., 2002; Rezende et al., 2015), pois depende do estado de relaxamento e baixo ruído elétrico para melhor definição da resposta (Beck et al., 2014; Sininger et al., 2018; Heil et al., 2020) .

Resultados similares são descritos na literatura quando mostram que os limiares eletrofisiológicos da RAEE são elevados em grupos de indivíduos com deficiência auditiva, independente da metodologia empregada (Picton et al., 2003; Komazec et al., 2010; Beck et al., 2014).

Outros estudos mostram que para detectar audição normal, a técnica dicótica múltipla da RAEE, realizadas neste estudo, ou seja, estimulação das duas orelhas e das quatro frequências simultaneamente é um método viável para estimar limiares auditivos que reduziriam substancialmente o tempo de registro em comparação com as técnicas convencionais de estímulo único (Herdman \& Stapells, 2001; Ghasemahmad \& Farahani, 2019).

Já é bem estabelecido de que indivíduos adultos com audição dentro dos padrões da normalidade, a média das frequências de 500, 1000 e $2000 \mathrm{~Hz}$, deve ser menor ou igual a $25 \mathrm{dBNA}$, entretanto, para limiares obtidos por medidas eletrofisiológicas ainda não há um padrão definido (Bucuvic \& Iório, 2011). É observado que os valores obtidos variam de acordo com a frequência pesquisada, sendo estes piores para as frequências graves e melhores para as frequências agudas (Silva et al., 2021).

Uma possível explicação para esse achado seria o resultado de uma dessincronização dos neurônios geradores da resposta, devido a um atraso no tempo de transmissão dos receptores cocleares e dos neurônios, o que resultaria numa diminuição na amplitude dos sinais registrados nas frequências graves, principalmente em $500 \mathrm{~Hz}$, tanto em indivíduos adultos como em crianças mais velhas (Ferraz et al., 2002; Komazec et al., 2010; Lachowska et al., 2012).

Devido a variabilidade de respostas na RAEE deste estudo, não foi possível estabelecer uma relação direta dos limiares auditivos obtidos por ambas as técnicas, sendo necessário mais estudos que envolvam casuísticas maiores, pois uma pesquisa realizada por Ahn et al. (2007) que compararam os resultados da ATL e RAEE em 105 indivíduos (168 orelhas, sendo 64 indivíduos do sexo masculino e 41 do sexo feminino) mostraram que os limiares da ATL e RAEE foram altamente correlacionados $(\mathrm{r}=0,96)$. Quando analisada de acordo com as frequências, os coeficientes de correlação foram 0,$94 ; 0,95 ; 0,94$ e 0,92 para 500, 1000, 2000 e $4000 \mathrm{~Hz}$, respectivamente. Concluíram que a ATL e RAEE mostraram resultados muito semelhantes e sugeriram que a RAEE poderia ser uma boa alternativa para medir os níveis de audição em lactentes e crianças, onde a ATL não é apropriada.

Komazec et al. (2010) avaliaram a confiabilidade da RAEE em determinar limiares em frequência específica para estabelecer uma correlação entre os limiares auditivos determinados pela ATL, bem como avaliar a confiabilidade da RAEE em determinar esse limiar com respeito ao grau e configuração da perda auditiva. O estudo incluiu 46 sujeitos ( 92 orelhas) que foram divididos em grupos baseados em seus níveis de deficiência auditiva e configuração audiométrica. A diferença entre 
limiares de RAEE e de ATL foram 4,1; 2,5; 4,4 e 4,2 dB em 500, 1000, 2000 e 4000Hz, respectivamente. O coeficiente de correlação entre os limiares auditivos determinados pela RAEE e pela ATL foi significante em sujeitos com todos os graus de perda auditiva e a diferença entre limiares auditivos foi menos de $10 \mathrm{~dB}$ em $85 \%$ dos sujeitos (variando de 4 dBNA para perda auditiva moderadamente severa a 7,2 dBNA para audição normal). Concluíram que a RAEE é um excelente método complementar para determinação do limiar auditivo, bem como na determinação das configurações audiométricas.

Assim, os resultados do presente estudo mostram que a variabilidade dos resultados obtidos na RAEE e o pequeno número da amostra podem ter interferido na baixa correlação entre as técnicas.

\section{Conclusão}

Os limiares auditivos obtidos pela RAEE foram detectados em todos os participantes do estudo e os valores foram superiores aos encontrados na ATL, entretanto é comum os resultados eletrofisiológicos serem superiores aos observados em exames comportamentais. Não houve correlação entre os limiares obtidos entre as técnicas para a maioria das frequências avaliadas, para indivíduos adultos normo-ouvintes. Desta forma, sugere-se a realização de pesquisas futuras com um maior tamanho amostral, adotando-se a mesma metodologia aplicada nesse estudo.

\section{Referências}

Ahn, J. H., Lee, H. S., Kim, Y. J., Yoon, T. H. \& Chung, J. W. (2007). Comparing pure-tone audiometry and auditory steady state response for the measurement of hearing loss. Otolaryngol Head Neck Surg, 136(6), 966-71.

Beck, R. M. O., Ramos, B. F., Grasel, S. S., Ramos, H. F., Moraes, B. B., Almeida, E. R. \& Bento, C. F. (2014). Comparative study between pure tone audiometry and auditory steady-state response in normal hearing subjects. Braz J Otorhinolaryngol, 80(1), 35-40.

Bucuvic, E. C. \& Iório, M. C. M. (2011). Resposta auditiva de estado estável. In: Bevilacqua, M. C., Martinez, M. A. N., Balen, A. S., Pupo, A. C., Reis, A. C. M. B. Frota S. (2 ed.), Tratado de Audiologia (pp. 197-215). São Paulo: Livraria Santos.

Dabbous, A. O., El-Shennawy, A. M., Medhat, M. M., Abdel-Latief, D. F. (2017). Narrow band CE-chirp stimulus in auditory steady state response threshold in normal hearers and patients with various degrees of sensorineural hearing loss. Hear Balance Commun, 15, $199-213$.

Dabbous, A.O., Koura, R. A. \& Hamdy, M. M. (2019). Comparison of thresholds estimation in adults with conductive hearing loss and normal hearing adults using auditory steady state response evoked by narrow band CE-chirps. Hear Balance Commun, 17, 18-26.

D’Haenens, W., Vinck, B. M., De Vel, E., Maes, L., Bockstael, A., Keppler, H., Philips, B., Swinnen, F. \& Dhooge, I. (2008). Auditory steady-state responses in normal hearing adults: a test-retest reliability study. Int J Audiol, 47(8), 489-98.

Estrela, C. (2018). Metodologia Científica: Ciência, Ensino, Pesquisa. (3a ed.), Artes Médicas.

Ferraz, O. B., Freitas, S. V. \& Marchiori, L. L. M. (2002). Análise das respostas obtidas por potenciais evocados auditivos de estado estável em indivíduos normais. Rev Bras Otorrinolaringol, 68(4), 480-6.

Ghasemahmad, Z., Farahani, S. (2019). Validity of correction factors applied to auditory steady-state responses (ASSRs) in normal hearing adults in chartr EP system. Eur Arch Otorhinolaryngol, 276, 2171-80.

Hei, N. \& Dong-Hee, L. (2020). Discrepancies in Hearing Thresholds between Pure-Tone Audiometry and Auditory Steady-State Response in NonMalingerers. Ear and Hearing, 41(3), 663-8.

Herdman, A. T. \& Stapells, D. R. (2001). Thresholds determined using the monotic and dichotic multiple auditory steady-state response technique in normalhearing subjects. Scand Audiol, 30(1), 41-9.

John, M. S. \& Picton, T. W. (2000). A Windows program for recording multiple auditory steady-state responses. Comput Methods Programs Biomed, 61(2), 125-50.

Komazec, Z., Lemajić-Komazec, S., Jović, R., Nadj, C., Jovancević, L. \& Savović, S. (2010). Comparison between auditory steady-state responses and puretone audiometry. Vojnosanit Pregl, 67 (10-11), 761-5.

Korczac, P., Smart, J., Delgado, R., Strobel, T. M. \& Bradford, C. (2012). Auditory steady-state responses. J Am Acad Audiol, 23(3), 146-70.

Lachowska, M., Surowiec, P., Morawski, K., Pierchała, K., \& Niemczyk, K. (2014). Second stage of universal neonatal hearing screening - a way for diagnosis and beginning of proper treatment for infants with hearing loss. Adv Med Sci, 59(1), 90-4.

Lins, O. G., Picton, T. W., Boucher, B. L., Durieux-Smith, A., Campagne, S. C., Moran, L. M., Perez-Abalo, M. C., Martin, V. \& Savio, G. (1996). Frequency-specific audiometry using steady-state responses. Ear Hear, 17(2), 81-96. 
Research, Society and Development, v. 10, n. 13, e174101320859, 2021

(CC BY 4.0) | ISSN 2525-3409 | DOI: http://dx.doi.org/10.33448/rsd-v10i13.20859

Picton, T. W., John, M. S., Dimitrijevic, A. \& Purcell, D. W. (2003). Human auditory steady-state responses. Int J Audiol, 42(4), 177-219.

Resende, L. M., Carvalho, S. A. S., Santos, T. S., Abdo, F. I., Ferreira, M. C. \& Tierra-Criollo, C. J. (2015). Auditory steady-state responses in school-aged children: a pilot study. J Neuroeng Rehabil, 12(1), 13.

Silva, D. P. C., Ribeiro, G. E. \& Montovani, J. C. (2021). Results of BAEP and Auditory Steady State Response in infants with and without UNHS failure. Distúrb Comun, 33(2): 339-47.

Sininger, Y. S., Hunter, L. L., Hayes, D., Roush, P. A., Uhler, K. M. (2018). Evaluation of Speed and Accuracy of Next-Generation Auditory Steady State Response and Auditory Brainstem Response Audiometry in Children with Normal Hearing and Hearing Loss. Ear Hear, 39(6): 1207-23.

Valdes, J. Lp., Perez-Abalo, M. C., Martin, V., Savio, G., Sierra C, Rodriguez, E. \& Lins, O. (1997). Comparison of statistical indicators for the automatic detection of $80 \mathrm{~Hz}$. auditory steady-state responses. Ear Hear, 18(5), 420-9. 